Editorial: Hospital Metropolitano

ISSN (impreso) 1390-2989 - ISSN (electrónico) 2737-6303

Edición: Vol. 28 No 3 (2020) Julio - Septiembre

DOI: https://doi.org/10.47464/MetroCiencia/vol28/3/2020/19-24

URL: https://revistametrociencia.com.ec/index.php/revista/article/view/82

Pág: 19-24

\title{
Ecografía transfontanelar en la enfermedad hipóxico isquémica del recién nacido. Presentación de un caso
}

\author{
Transfontanellar ultrasound in hypoxic disease \\ newborn ischemic. Presentation of a case
}

\author{
Dayana Torres' ${ }^{(D)}$, José Sosa ${ }^{2}$ iD, Gonzalo Dueñas ${ }^{3}$ \\ Médicos Residentes del Posgrado de Imagenología, Universidad San Francisco de Quito, Ecuador ${ }^{1,2}$ \\ Neurorradiólogo, Hospital Metropolitano Quito, Ecuador ${ }^{3}$
}

Recibido: 10/4/2020 Aceptado: 26/6/2020 Públicado: 01/09/2020

\begin{abstract}
RESUMEN
La enfermedad hipóxico-isquémica constituye una de las principales causas de morbi-mortalidad neurológica en el recién nacido. Las diferentes adaptaciones vasculares a la hipoxia tanto en el neonato pretérmino como en niño a término hacen que su presentación en neuroimagen, sobre todo en el ultrasonido (US) sea caracterizable según el territorio afectado y el momento de la enfermedad. El ultrasonido se ha convertido en una poderosa herramienta para la evaluación del recién nacido con sospecha de EHI, y el patrón de las lesiones tiene importantes implicaciones en el tratamiento y en el pronóstico neurológico a largo plazo. A continuación, presentamos el caso de un recién nacido masculino, prematuro extremo, que requirió reanimación cardiopulmonar avanzada en el nacimiento y que además presento dos episodios de parada cardiorrespiratoria en el segundo y tercer día de vida, en el cual se llegó al diagnóstico con patrones ecográficos característicos de lesión isquémica y además se detalla la evolución de los hallazgos en el tiempo.
\end{abstract}

Palabras claves: Enfermedad hipóxico-isquémica, ultrasonido transfontanelar, matriz germinal, leucomalacia periventricular.

\section{ABSTRACT}

Hypoxic-ischemic disease is one of the main causes of neurological morbidity and mortality in the newborn. The different vascular adaptations to hypoxia in both the preterm and term infants make their presentation on neuroimaging, especially on ultrasound (US), characterizable according to the affected territory and the time of the disease. Ultrasound has become a powerful tool for evaluating the newborn with suspected IBD, and the pattern of the lesions has important implications for treatment and long-term neurological prognosis. Next, we present the case of a male newborn, extremely premature, who required advanced cardiopulmonary resuscitation at birth and who also presented two episodes of cardiorespiratory arrest on the second and third day of life, in which the diagnosis was reached with patterns sonographic characteristics of ischemic injury and also the evolution of the findings over time.

Keywords: Hypoxic-ischemic disease, transfontanelar ultrasound, germ matrix, periventricular leukomalacia.

Dayana Torres:

José Sosa:

Gonzalo Dueñas:

\section{IDs Orcid}

https://orcid.org/0000-0002-0836-1420

https://orcid org/0000-0002-8021-2702

https://orcid.org/0000-0003-2272-1397

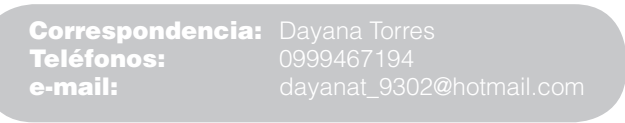




\section{INTRODUCCIÓN}

La EHI continúa siendo una causa importante de muerte y discapacidad del desarrollo neurológico en recién nacidos ${ }^{1,2}$. La prevalencia de $\mathrm{EHI}$ se estima entre dos y cuatro por 1000 nacimientos a término vivos $^{1-3}$. La EHI es una de las causas más comunes de parálisis cerebral y otros déficits neurológicos graves en niños. 67 La alteración del flujo sanguíneo cerebral puede deberse a un compromiso cardíaco y vascular fetal, que se produce en el útero o postnatalmente. Dicha interrupción puede ser causada por factores fetales (hemorragia feto-materna, trombosis fetal y bradicardia fetal), perfusión placentaria inadecuada (hipotensión materna, pre eclampsia, enfermedad vascular crónica, desprendimiento prematuro de placenta), oxigenación materna deteriorada o circulación umbilical interrumpida (cordón nucal apretado, prolapso del cordón). La asfixia posnatal puede ser el resultado de una enfermedad de membrana hialina grave, neumonía, aspiración de meconio o anomalías cardíacas congénitas que causan insuficiencia pulmonar neonatal o hipotensión. Independientemente de la causa de la asfixia, el compromiso cardíaco y vascular fetal definitivo conduce a una disminución del flujo sanguíneo cerebral, pérdida de autorregulación cerebral y muerte celular neuronal2,7,9.

El patrón de lesión en las imágenes del cerebro tiene implicaciones cruciales en la terapia y los resultados predichos del desarrollo neurológico. El US se ha vuelto cada vez más efectivo para determinar el patrón, el momento y el alcance de la lesión en la EHI, así como para diferenciar estos hallazgos de una serie de diagnósticos que pueden dar como resultado un cuadro clínico similar-8,12-13. Los estudios periódicos de ultrasonido sobre el curso de un paciente con la enfermedad pueden definir la evolución de los hallazgos desde la fase aguda hasta la crónica, además de identificar cualquier complicación de la terapia $^{16,19,20}$.

EI US trans-fontanelar sigue siendo el primer estudio que se realiza al neonato con sospecha de EHI, además tiene los beneficios adicionales de la fácil portabilidad, la no necesidad de sedación del paciente y un costo relativamente bajo en comparación con otras modalidades de imagen. Sin embargo, pese a todos estos beneficios, la sensibilidad del estudio es baja $(50 \%)$, por lo que un estudio negativo, no debe ser considerado como excluyente de enfermedad, si las condiciones clínicas lo indican una resonancia magnética debe ser solicitada para una mejor evaluación ${ }^{2-4}$. Dentro del espectro de la EHI existen 2 signos que son fácilmente evaluables por ultrasonografía transfontanelar: la hemorragia de la matriz germinal (EMG) y la leucomalacia periventricular (LPV), signos en los que nos centraremos en este manuscrito ${ }^{2,14,13}$.
La prevalencia general de hemorragia intraventricular en recién nacidos prematuros que pesan menos de $2000 \mathrm{~g}$ es aproximadamente del 25\%, y en la mayoría de los casos esta hemorragia ocurre dentro de las primeras 24 horas de vida ${ }^{11-14}$. La hemorragia intraventricular tiene como etiología principal la hemorragia de la matriz germinal, la matriz germinal es un área celular especializada a partir de la cual se originan las neuronas y células gliales que migraran hacia las diferentes áreas cerebrales durante el desarrollo del neonato1,6,9,12. La matriz germinal se encuentra tapizando las paredes de los ventrículos laterales y posteriormente involuciona y queda un área remanente en el surco caudotalamico, área comprendida entre el núcleo caudado y tálamo, que en términos de cribado para descartar hemorragia de la matriz germinal es un área que no debe pasarse por alto en el examen ecográfico ${ }^{1-3}$.

La matriz germinal tiene una disposición vascular especial respecto a los vasos sistémicos, estos son más grandes y están recubiertos solo por endotelio vascular, sin muscular y colágeno de soporte; también la gran cantidad de mitocondrias visualizadas dentro de las células (5 veces más que otras células) denotan la gran cantidad de requerimiento de metabolismo oxidativo, lo cual las hace más sensibles a los cambios de oxigenación $n^{5,6}$.

\section{REPORTE DEL CASO}

En la emergencia del Hospital Metropolitano se recibe una paciente gestante cursando embarazo de 25.5 semanas, quien acude por presentar dolor abdominal tipo cólico, EVA 10/10, de 4 horas de evolución, además presenta modificación cervical: $9 \mathrm{~cm}$ de dilatación, 90 \% de borramiento, y presiones arteriales elevadas.

Se recibe por cesárea de emergencia a RN masculino vivo, APGAR: 5-8. Se utilizó fórceps de Simpson para su extracción, a la valoración inicial presenta: pobre tono muscular, flácido, llanto muy débil, cianosis generalizada, frecuencia cardiaca menor a 100. Se utilizó C-PAP por 1,5 minutos, sin mejoría pese al uso de pasos iniciales de reanimación por lo que se decide intubación endotraqueal y se traslada al área de neonatología en cuna de calor radiante. A su ingreso al área de neonatología se recibe recién nacido activo y reactivo al manejo. Se realiza ecografía transfontanelar en las primeras 24 horas de vida; la cual no reveló hallazgos significativos. (Figura 1 y 2). 


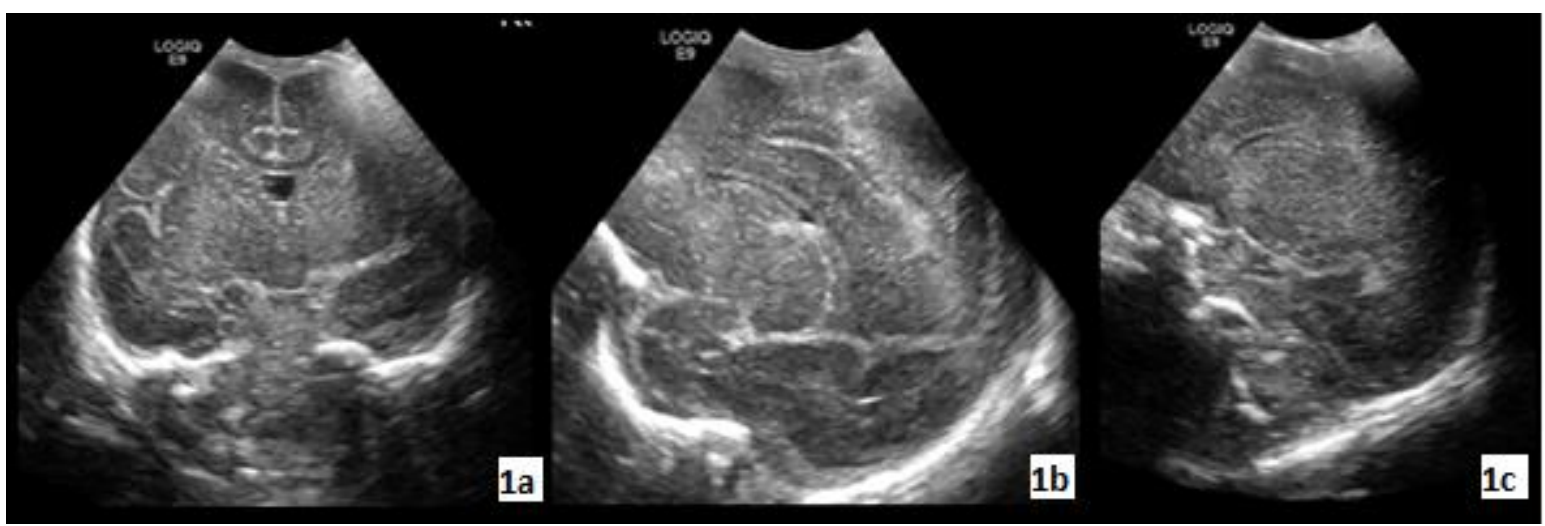

Figura 1. Ultrasonido: Transfontanelar. (1a) Corte coronal de ecografía transfontanelar en la que se observa lóbulos temporales, frontales, tálamo, ventrículos laterales, cavum vergae de características habituales. (1b) Corte para sagital derecho en la que se observa ventrículo lateral, tálamo, surco caudotalamico, lóbulo temporal y frontal de características normales. (1c) Corte para sagital izquierdo en la que se observa ventrículo lateral, tálamo, surco caudotalamico, lóbulo temporal y frontal de características normales.

Fuente: Los autores

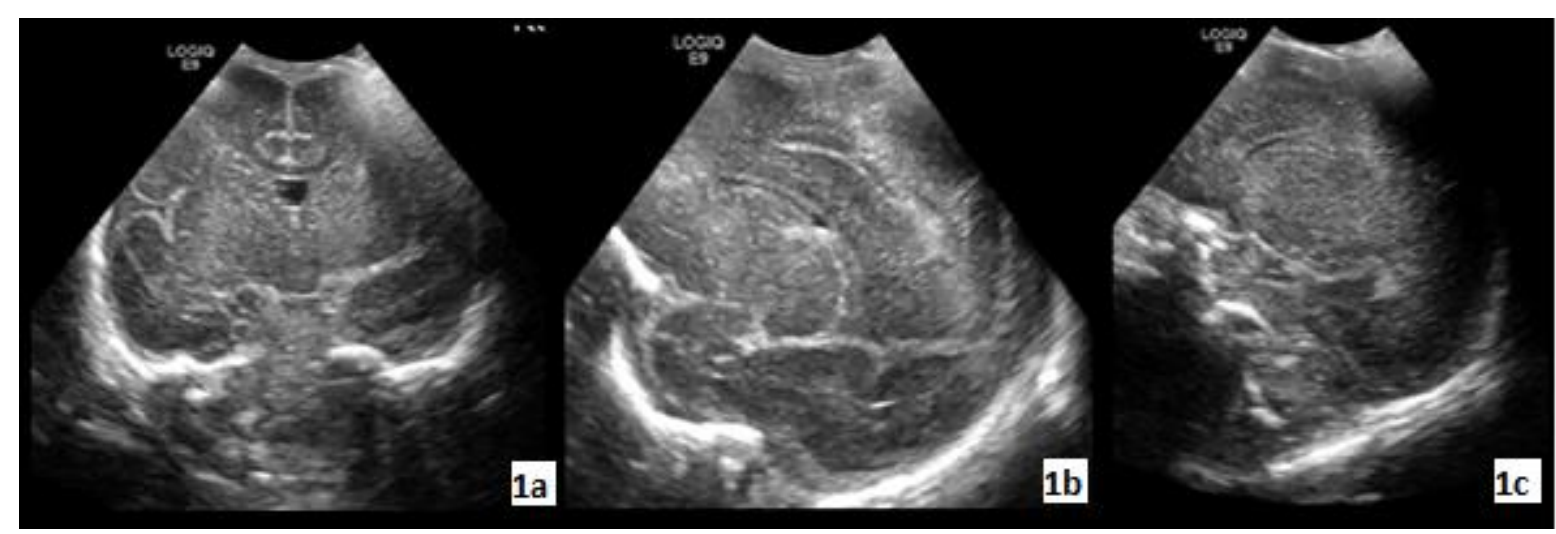

Figura 2. Ecografía Espectral. (2a) Doppler espectral de arteria cerebral media, se observa onda fásica de características normales. (2b) Doppler espectral de arteria cerebral media en la cual se demuestra un índice de resistencia de parámetros normales.

Fuente: Los autores

Posteriormente realiza parada cardio respiratoria por 2 ocasiones el segundo y tercer día de vida, con una duración de aproximada de 2 y 1 minuto respectivamente. Después del evento inicialmente se presentó hipo activo al manejo, además presenta evento sugestivo de convulsiones con chupeteo, motivo por el cual se solicita nuevamente ecografía transfontane- lar en la que podemos encontrar signos de hemorragia de la matriz germinal e intraventricular derecha asociada a dilatación del ventrículo e incremento de la ecogenicidad de la sustancia blanca periventricular ipsilateral; hallazgos típicos de la EHI. (Figura 3, 4 y 5$)$ 


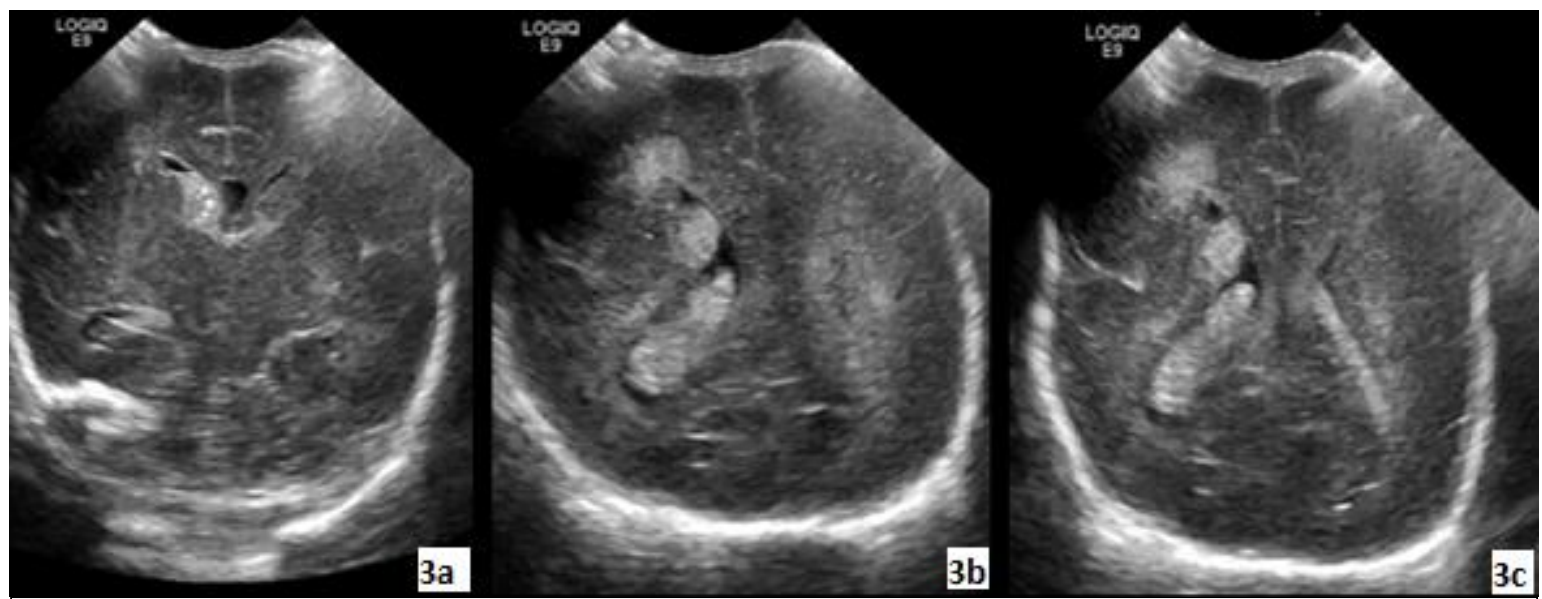

Figura 3. Ultrasonografía Transfontanelar. (3a) Corte coronal de ultrasonografía en la que se observa área ecogenica en el surco caudo talámico derecho que se impresiona inferior al ventrículo cerebral derecho y dilatación del cuerno temporal derecho. (3b) Corte coronal en la que se observa ventrículo derecho dilatado con áreas ecogenicas redondeadas correspondientes a coágulos sanguíneos, en el área periventricular derecha frontal se visualiza área ecogénica correspondiente a zona de leucomalacia. (3c). Corte coronal con hallazgos similares de ventriculomegalia y área ecogenica periventricular frontal derecha secundarios a hemorragia por hipoxia e isquemia cerebral.

Fuente: Los autores

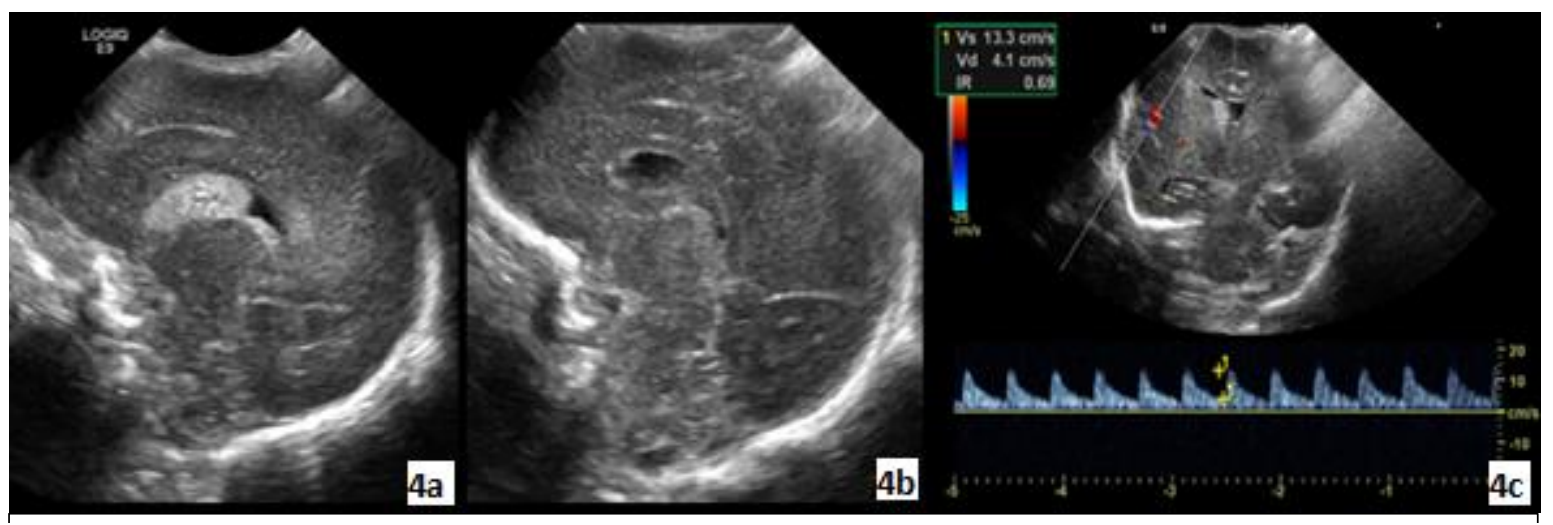

Figura 4. Ultrasonografía en escala de grises y doppler espectral. (3a) Corte para sagital derecho en la que se observa área ecogenica en el surco caudotalamico e intraventricular este último correspondiente a coagulo. (4b) Corte para sagital Izquierdo sin alteraciones. (4c) Doppler espectral de arteria cerebral media derecha en la que se observa un IR normal.

Fuente: Los autores

Posteriormente se realizó seguimiento ecográfico para visualizar los cambios morfológicos de las lesiones antes mencionadas, en las que se observan signos regresión del coagulo intraventricular dere- cho, y signos típicos de leucomalacia periventricular con degeneración quística como secuela de hemorragia secundario a hipoxia e isquemia. (Figura 5) 


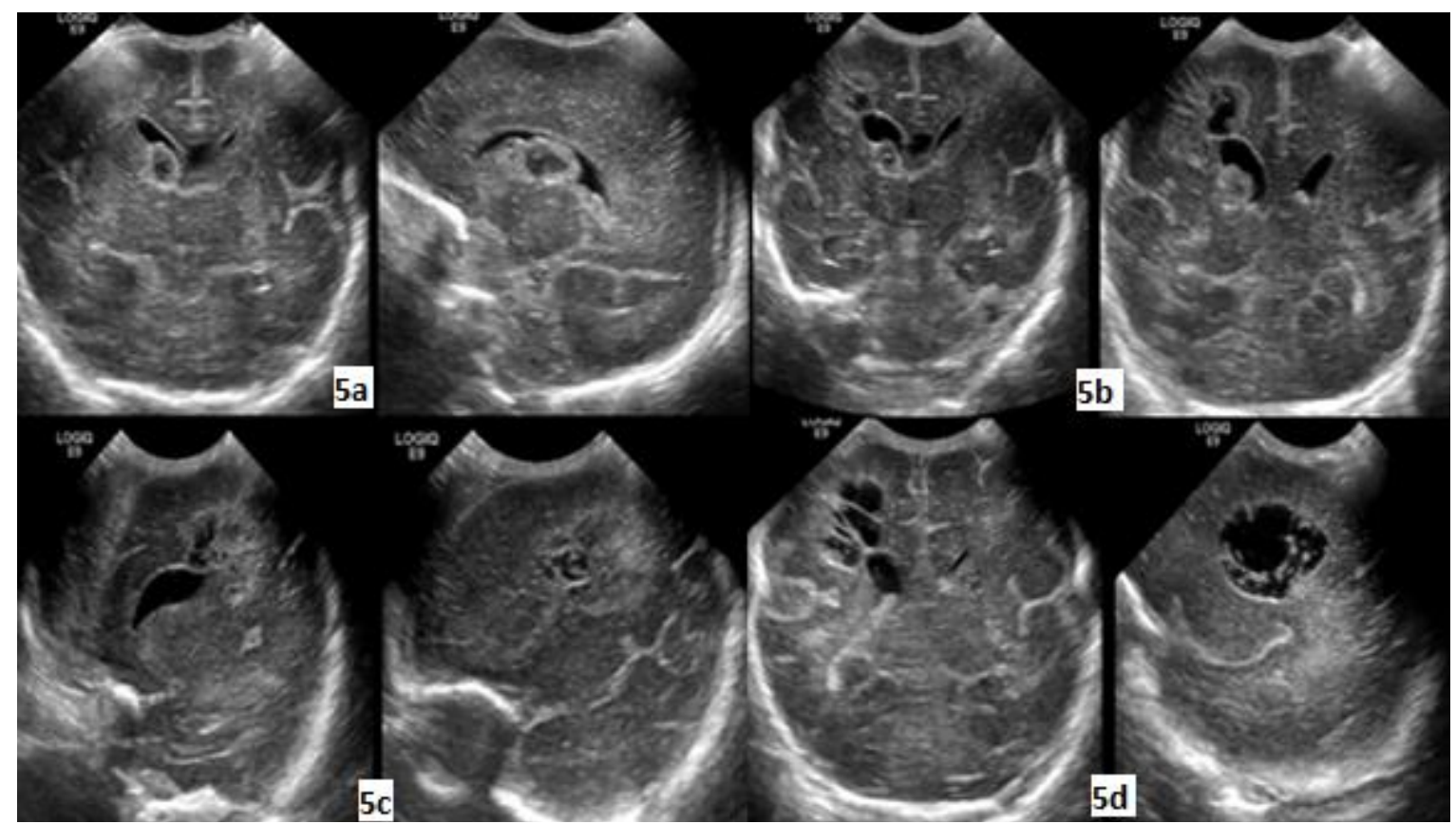

Figura 5. Ultrasonografía Transfontanelar. (5a) cortes coronal y parasagital en la que se observa degeneración quística del coagulo en el surco caudotalámico secundario a hemorragia de la matriz. (5b) cortes coronales en la que se observan estigmas de enfermedad de sustancia blanca periventricular con áreas de leucomalacia quística periventricular. (5c) cortes parasagitales derechos en las que se observa áreas de enfermedad de sustancia blanca periventricular con leucomalacia quística. (5d) cortes coronal y parasagital derechos en los que se observa en el área periventricular áreas de leucomalacia quística.

Fuente: Los autores

\section{DISCUSIÓN}

La encefalopatía hipoxico isquémica es uno de los principales cuadros con importante morbilidad y mortalidad infantil, las causas que llevan hacia la hipoxia pueden ser numerosas tanto en la etapa prenatal y posnatal ${ }^{15}$, la fisiopatología no está del todo comprendida, pero se cree que la falta de flujo sanguíneo y oxigenación tisular lleva a una alteración en la autorregulación vascular cerebral que condiciona hipoxia y posterior isquemia cerebral con daño del parénquima sobre todo en el neonato pretérmino ${ }^{15-18}$.

Las áreas cerebrales vulnerables a la injuria varían en función de múltiples factores, tales como el grado de madurez cerebral, la severidad de la injuria o la duración del mismo. Así, no se lesionarán las mismas áreas en un paciente prematuro que otro a término o en casos de hipoxia de corta duración frente a hipoxia prolongada. En la población pediátrica la injuria hipóxico-isquémico debe tener una duración de al menos 10-15 minutos para producir secuelas $6,7,12$.

Una de las manifestaciones tempranas de hipoxia e isquemia es la hemorragia de la matriz germinal y posteriormente como consecuencia de la misma tenemos enfermedad de la sustancia blanca que se expresa como leucomalacia periventrular, las dos entidades pueden detectarse fácilmente en la eco- grafía transfontanelar y se puede clasificar dependiendo de los lugares y repercusión sobre el tejido circundante hablando de la hemorragia de la matriz germinal, y el grado de enfermedad de la sustancia blanca que se expresa como degeneración quística o gliosis dependiendo de la región afectada en la leucomalacia periventricular ${ }^{14}$.

En función de lo mencionado anteriormente se han descrito los siguientes grados de la hemorragia de la matriz germinal:

- Grado I: Hemorragia subependimaria sin o con extensión mínima hacia los ventrículos.

- Grado II: Hemorragia de la matriz germinal que se extiende hacia los ventrículos, sin dilatación de los mismos.

- Grados III: Hemorragia de la matriz germinal que se extiende hacia los ventrículos y presentan dilatación de los mismos.

- Grado IV: Infarto hemorrágico periventricular.

Posterior a un sangrado de matriz germinal de grado III o IV, el área de infarto hemorrágico periventricular degenera hacia enfermedad de sustancia blanca, patología conocida como leucomalacia periventricular $^{19,20}$. La leucomalacia periventricular (LPV) es 
la forma más común de daño cerebral hipoxémico en los niños pequeños. En la LPV generalmente hay antecedentes de asfixia o hipoxia. Esto da como resultado un daño hipóxico-isquémico en la región del cerebro alrededor de los ventrículos laterales, un área particularmente vulnerable ya que se encuentra en una zona fronteriza entre los suministros arteriales en los pacientes pre termino sobretodo ${ }^{9,15,16,20}$.

Inicialmente la exploración ecográfica puede ser normal. Sin embargo, en dos semanas la sustancia blanca periventricular aumenta de ecogenicidad hasta que es mayor que la del plexo coroideo adyacente. Este aumento de ecogenicidad está causado por el edema ocasionado por el infarto y ocasionalmente por hemorragia ${ }^{15,16}$.

De igual forma se han descrito varios grados de leucomalacia periventricular:

- GRADO I: Incremento de la ecogenicidad periventricular que persiste más de 7 días.

- GRADO II: Desarrollo de pequeños quistes periventriculares.

- GRADO III: Áreas de mayor ecogenicidad periventricular + quistes periventriculares extensos en la región occipital y frontoparietal.

- GRADO IV: Ecogenicidad periventricular en la materia blanca profunda + quistes subcorticales extensos ${ }^{14,19,20}$.

\section{CONCLUSIÓN}

La Enfermedad Hipoxico Isquémica es una patología que representa una causa importante de morbilidad y mortalidad en recién nacidos. El ultrasonido es una valiosa herramienta de detección en el diagnóstico y manejo de neonatos con encefalopatías, con experiencia se puede reconocer signos tempranos como hemorragia de la matriz germinal y clasificarla según su comportamiento, así como también realizar seguimiento cuando existe enfermedad de la sustancia blanca que se expresa como leucomalacia periventricular.

El radiólogo debe estar entrenado para detectar estos signos, ya que las causas que los producen son varias tanto en la vida prenatal y posnatal y finalmente las decisiones médicas no deben posponerse debido a la falta de imágenes de resonancia magnética cuando los hallazgos de US son consistentes con el cuadro clínico.

\section{REFERENCIAS BIBLIOGRÁFICAS}

1. Hypoxic-Ischemic Brain Injury: Imaging Findings from Birth to Adulthood Benjamin Y. Huang and Mauricio Castillo RadioGraphics 2008

\section{$28: 2,417-439$}

2. Salas J, Tekes A, Hwang M, Northington F, J, Huisman T, A, G, M: Head Ultrasound in Neonatal Hypoxic-Ischemic Injury and Its Mimickers for Clinicians: A Review of the Patterns of Injury and the Evolution of Findings Over Time. Neonatology 2018;:185-197.

3. Neonatal Hypoxic-Ischemic Encephalopathy: Multimodality Imaging Findings Christine P. Chao, Christopher G. Zaleski, and Alice C. Patton RadioGraphics 2006 26:suppl_1, S159-S172

4. Moral Y, Robertson NJ, Goñi-de-Cerio F, Alonso-Alconada D. Hipoxia-isquemia neonatal: bases celulares y moleculares del daño cerebral y modulación terapéutica de la neurogénesis. Rev Neurol 2019;68 (01):23-36

5. Novoa P, José M, Milad A, Marcela, Fabres B, Jorge, Fasce C, Juan A, Toso M, Paulina A, Arriaza O, Manuel, Gandolfi E, Carolina, Samamé $M, M^{a}$ Margarita, \& Aspillaga M, Carlos. (2012). Consenso sobre manejo integral del neonato con encefalopatía hipóxico isquémica. Revista chilena de pediatría, 83(5), 492-501.

6. Staneva KN, Bartolomaeus G. Sonographische Diagnose der periventrikulären Leukomalazie [Ultrasound diagnosis of periventricular leukomalacia]. Z Geburtshilfe Neonatol. 1997;201(2):4448.

7. Periventricular leukomalacia: radiologic diagnosis. O Flodmark, E H Roland, A Hill, and M F Whitfield Radiology 1987 162:1, 119-124.

8. Kurinczuk JJ, White-Koning M, Badawi N: Epidemiology of neonatal encephalopathy and hypoxic-ischaemic encephalopathy. Early Hum Dev 2010; 86: 329-338.

9. Daneman A, Epelman M, Blaser S, Jarrin JR: Imaging of the brain in full-term neonates: does sonography still play a role? Pediatr Radiol 2006; 36: 636-646.

10. Martinez-Biarge M, Bregant T, Wusthoff CJ, Chew AT, Diez-Sebastian J, Rutherford MA, Cowan FM: White matter and cortical injury in hypoxic-ischemic encephalopathy: antecedent factors and 2-year outcome. J Pediatr 2012; 161: 799-807.

11. Ferriero DM. Neonatal brain injury. N Engl J Med 2004;351:1985-1995.

12. Periventricular leukomalacia in combination with intraventricular hemorrhage: sonographic features and sequelae. E G Grant, D Schellinger, Y Smith, R H Uscinski American Journal of Neuroradiology May 1986, 7 (3) 443-447.

13. Hernández Velázquez, Norbelis, Landrove Borjas, Idalmis, \& Andrés Matos, Andrés. (2014). Evaluación neurológica en recién nacidos con asfixia al nacer. Correo Científico Médico, 18(3), 457-468

14. M. Martínez-Biarge, D. Blanco, A. García-Alix, S. Salas, Seguimiento de los recién nacidos con encefalopatía hipóxico-isquémica, Anales de Pediatría, Volume 81, Issue 1, 2014, Pages 52.e1-52.e14, ISSN 1695-4033.

15. Ahya KP, Suryawanshi P. Neonatal periventricular leukomalacia: Current perspectives. Research and Reports in Neonatology. 2018;8:1-8.

16. Deng, W, Pleasure, $J$ and Pleasure, D (2008). Progress in Periventricular Leukomalacia. Arch Neurol 65(10): 1291-1295, DOI: https:// doi.org/10.1001/archneur.65.10.1291 PMid: 18852342; PMCid: PMC2898886.

17. Chao, CP, Zaleski, CG and Patton, AC (2006). Neonatal Hypoxic-Ischemic Encephalopathy: Multimodality Imaging Findings. RadioGraphics 26: S159-S172, DOI: https://doi.org/10.1148/rg.26si065504 PMid: 17050513

18. Back, SA (2014). Cerebral White and Gray Matter Injury in Newborns New Insights into Pathophysiology and Management. Clin Perinatol 41: 1-24, DOI: https://doi.org/10.1016/j.clp.2013.11.001 PMid: 24524444

19. de Vries, LS, Eken, $P$ and Dubowitz, LM (1992). The spectrum of leukomalacia using cranial ultrasound. Behav Brain Res 49(1): 1-6, DOI: https://doi.org/10.1016/S0166-4328(05)80189-5

20. Van Camp L, Steyaert L. Cystic Periventricular Leukomalacia: A Condition that Became Uncommon in the Premature Neonate, Diagnosed on Transcranial Ultrasound. Journal of the Belgian Society of Radiology. 2016;100(1):8. DOI: http://doi.org/10.5334/jbr-btr. 1000

Como citar este artículo: Torres D, Sosa J, Dueñas G. Ecografía transfontanelar en la enfermedad hipóxico isquémica del recién nacido. Presentación de un caso. MC [Internet]. 1 de septiembre de 2020;28(3):19-24. https://doi.org/10.47464/MetroCiencia/vol28/3/2020/19-24 